\title{
RATIONAL POINTS ON CERTAIN DEL PEZZO SURFACES OF DEGREE ONE
}

\author{
MACIEJ ULAS \\ Jagiellonian University, Institute of Mathematics, Reymonta 4, 30-059 Kraków, Poland \\ e-mail:Maciej.Ulas@im.uj.edu.pl
}

(Received 8 October 2007; revised 21 January 2008; accepted 1 February 2008)

\begin{abstract}
Let $f(z)=z^{5}+a z^{3}+b z^{2}+c z+d \in \mathbb{Z}[z]$ and let us consider a del Pezzo surface of degree one given by the equation $\mathcal{E}_{f}: x^{2}-y^{3}-f(z)=0$. In this paper we prove that if the set of rational points on the curve $E_{a, b}: Y^{2}=X^{3}+135(2 a-$ 15) $X-1350(5 a+2 b-26)$ is infinite then the set of rational points on the surface $\mathcal{E}_{f}$ is dense in the Zariski topology.

2000 Mathematics Subject Classification. Primary 11D25, 11D41; Secondary $11 \mathrm{G} 052$.
\end{abstract}

1. Introduction. A projective and geometrically irreducible surface $S$ is called a del Pezzo surface if its anti-canonical class $K_{S}$ is ample. The degree of del Pezzo surface is the self-intersection number of its canonical class: $\operatorname{deg} S=K_{S}^{2}$. As we know, the number $\operatorname{deg} S$ is a positive integer, and in fact we have the inequality $1 \leq \operatorname{deg} S \leq 9$. Geometrically, smooth del Pezzo surfaces are obtained by blowing up $d \leq 8$ points in general position in $\mathbb{P}^{2}$. The singular ones are blow-ups of $\mathbb{P}^{2}$ in special configurations of points or in infinitely near points. A detailed study of geometric and arithmetic properties of del Pezzo surfaces can be found in [6].

Many interesting arithmetic questions are connected with the class of del Pezzo surfaces. As such surfaces are geometrically rational (they are rational over the field $\mathbb{C}$ ), it is especially interesting to look at problems concerning the question about density (in Zariski topology) of $k$-rational points, where $k$ is a fixed finite extension of the field of rational numbers. From the arithmetic and geometric points of view higher degree del Pezzo surfaces are simpler than low degree ones. For example, it turns out that in case $\operatorname{deg} S=5$ the surface $S$ is always $k$-rational. In case $\operatorname{deg} S \leq 4$ situation is more complicated. Indeed, there are many examples of singular del Pezzo surfaces of degree four that are unirational over $\mathbb{Q}$, but not rational.

In this paper we are interested in the problem of existence of rational points on del Pezzo surface of degree one given by the equation

$$
\mathcal{E}_{f}: x^{2}-y^{3}-f(z)=0,
$$

where $f(z)=z^{5}+a z^{3}+b z^{2}+c z+d \in \mathbb{Z}[z]$. It is clear that after a suitable change of coordinates each surface given by the equation $p x^{2}+q y^{3}+g(z)=0$, where $p, q \in \mathbb{Q} \backslash$ $\{0\}, g \in \mathbb{Q}[z], \operatorname{deg} g=5$ or $\operatorname{deg} g=6$ and $g$ has a rational root and can be transformed to the surface $\mathcal{E}_{f}$ for a suitably selected $f \in \mathbb{Z}[z]$ of degree five.

Now, if we fix $z \in \mathbb{Q}$ such that $f(z) \neq 0$ then the curve $\mathcal{E}_{f(z)}$ is an elliptic curve. So, in a natural way, we can consider $\mathcal{E}_{f}$ as on an elliptic surface where $z$-line is a base curve 
of the fibration. In our case $\mathcal{E}_{f}$ is an isotrivial elliptic surface with $j$-invariant $j\left(\mathcal{E}_{f}\right)=0$. In recent years several papers concerning the density of points in certain classes of isotrivial elliptic surfaces have appeared. Interested readers can take a look at papers $[1,4,5,7,8]$.

In Section 2 we prove Theorem 2.1 which says that if the set of rational points on the curve

$$
E_{a, b}: Y^{2}=X^{3}+135(2 a-15) X-1350(5 a+2 b-26)
$$

is infinite then rational points on the surface $\mathcal{E}_{f}$ are dense in Zariski topology. We also show that if $t$ is a transcendental parameter then, under the same assumption on the curve $E_{a, b}$, the set of $\mathbb{Q}[t]$-points on the surface

$$
\mathcal{E}_{f}: x^{2}-y^{3}-f(z)=t,
$$

which is treated over the field of rational functions $\mathbb{Q}(t)$, is infinite.

In Section 3 as well we show that for every pair $a, b$ of non-zero rational numbers the set of rational points on the surface

$$
S: x^{2}+a y^{5}-z^{6}=b
$$

(which is not a del Pezzo surface) is infinite.

2. Rational points on the surface $\mathcal{E}_{f}$. Let $f(z)=z^{5}+a z^{3}+b z^{2}+c z+d \in \mathbb{Z}[z]$ and let us consider the surface

$$
\mathcal{E}_{f}: x^{2}-y^{3}-f(z)=0 .
$$

For a given $z \in \mathbb{Q}$ denote the curve $Y^{2}=X^{3}+f(z)$ by $E_{z}$. Let us recall how the torsion part of the curve $E_{z}$ looks like with a fixed $z \in \mathbb{Q}[\mathbf{9}$, p. 323]. If $f(z)=1$, then Tors $E_{z} \cong \mathbb{Z} / 6 \mathbb{Z}$. If $f(z) \neq 1$ and $f(z)$ is a square in $\mathbb{Q}$, then Tors $E_{z}=$ $\{\mathcal{O},(0, \sqrt{f(z)}),(0,-\sqrt{f(z)})\}$. In the case that $f(z)=-432$ we have Tors $E_{z}=\{\mathcal{O}$, $(12,36),(12,-36)\}$. If $f(z) \neq 1$ and $f(z)$ is a cube in $\mathbb{Q}$, then Tors $E_{z}=$ $\{\mathcal{O},(-\sqrt[3]{f(z)}, 0)\}$. In the remaining cases we have Tors $E_{z}=\{\mathcal{O}\}$. The above have two important consequences. If $f$ does not have multiple roots then there are only finitely many rational numbers $z$ such that the curve $E_{z}$ has non-trivial torsion points. Indeed, because every curve $f(z)=\lambda v^{i}$, where $i=2,3,6$ and $\lambda \in\{1,-432\}$, is of genus $\geq 2$, our observation is an immediate consequence of Faltings Theorem [3] (of course if $i=0$ then the equation $f(z)=\lambda$ has at most five rational roots). Furthermore, if there is a rational base change $z=\psi(t)$ such that on the surface $\mathcal{E}_{\text {fo } \psi}$ we have the section $\sigma=(x, y) \in \mathbb{Q}(t) \times \mathbb{Q}(t)$ with $x y \neq 0$, then $\sigma$ is a non-torsion section. It is clear that we have the same conclusion in the case when $f$ has a rational multiple root and $f(\psi(t))$ is a non-constant sixth power free element of $\mathbb{Q}(t)$.

Now we are ready to prove the following:

THEOREM 2.1. Let $f(z)=z^{5}+a z^{3}+b z^{2}+c z+d \in \mathbb{Z}[z]$ and consider the surface given by the equation $\mathcal{E}_{f}: x^{2}-y^{3}-f(z)=0$. Then

(1) if $f$ has multiple roots over $\mathbb{C}$ then there exists a rational change of base $z=\psi(t)$ such that there is a non-torsion section on elliptic surface $\mathcal{E}_{\text {fow. }}$. In this case the set of rational points on $\mathcal{E}_{f}$ is Zariski dense. 
(2) if $f$ does not have multiple roots and the set of rational points on the curve $E_{a, b}: Y^{2}=X^{3}+135(2 a-15) X-1350(5 a+2 b-26)$ is infinite then the set of rational points on the surface $\mathcal{E}_{f}$ is Zariski dense.

Proof. (1) If polynomial $f$ has a rational multiple root then without loss of generality we can assume that $f(z)=z^{2}\left(z^{3}+a^{\prime} z^{2}+b^{\prime} z+c^{\prime}\right)$ for certain integers $a^{\prime}, b^{\prime}$ and $c^{\prime}$. For the proof of our theorem, it will be convenient to work with the surface $\mathcal{E}_{f}^{\prime}$ given by the equation

$$
\mathcal{E}_{f}^{\prime}: F^{\prime}(X, Y, Z):=X^{2}-Z Y^{3}-\left(Z^{3}+a^{\prime} Z^{2}+b^{\prime} Z+c^{\prime}\right)=0,
$$

which is birationally equivalent to $\mathcal{E}_{f}$ by the mapping $(x, y, z)=(Z X, Z Y, Z)$ with the inverse $(X, Y, Z)=(x / z, y / z, z)$. In order to find the rational curve on $\mathcal{E}_{f}^{\prime}$ we use method of indetermined coefficients. So, suppose that $X=Z^{2}+p Z+q, Y=Z+t$, where $t$ is transcendental parameter and we are looking for $p, q \in \mathbb{Q}(t)$. For $X, Y$ defined this way we obtain

$$
F^{\prime}(X, Y, Z)=f_{0}+f_{1} Z+f_{2} Z^{2}+f_{3} Z^{3},
$$

where

$$
\begin{array}{ll}
f_{0}=-c^{\prime}+q^{2}, & f_{1}=-b^{\prime}+2 p q-t^{3}, \\
f_{2}=-a^{\prime}+p^{2}+2 q-3 t^{2}, & f_{3}=-1+2 p-3 t .
\end{array}
$$

Let us note that the system of equations $f_{2}=f_{3}=0$ has exactly one solution in $\mathbb{Q}(t)$ given by

$$
p=\frac{1+3 t}{2}, \quad q=\frac{-1+4 a^{\prime}-6 t+3 t^{2}}{8}
$$

For $p$ and $q$ defined in this way we can now solve the equation $f_{0}+f_{1} Z=0$ according to $Z$,

$$
Z=-\frac{9 t^{4}-36 t^{3}+6\left(4 a^{\prime}+5\right) t^{2}-12\left(4 a^{\prime}-1\right) t+16 a^{\prime 2}-8 a^{\prime}-64 c^{\prime}+1}{8\left(t^{3}-15 t^{2}+3\left(4 a^{\prime}-3\right) t+4 a^{\prime}-8 b^{\prime}-1\right)}=: \psi(t) .
$$

Therefore, on the surface $\mathcal{E}_{f \circ \psi}$ we have the section $\sigma=(x, y)=\left(\psi(t)\left(\psi(t)^{2}+p \psi(t)+\right.\right.$ $q), \psi(t)+t$ ), where $p, q$ are given by (2.1). With the help of computer we find that $f(\psi(t))$ is a non-constant and sixth power free element of $\mathbb{Q}(t)$, thus, from the remark presented at the beginning of this section, the section $\sigma$ is a non-torsion section. After the base change we get infinitely many sections (corresponding to $m \sigma$ ), each with infinitely many rational points. That means that each section is included in the Zariski closure, say $\mathcal{R}$, of the set of rational points. Because this closure consists of only finitely many components, it has dimension two, and as the surface is irreducible, $\mathcal{R}$ is the whole surface. Thus the set of rational points on $\mathcal{E}_{f \circ \psi}$ is dense. The map from the $\mathcal{E}_{f \circ \psi}$ to the $\mathcal{E}_{f}$ is dominant, so the set of rational points on the surface $\mathcal{E}_{f}$ is also dense.

In the case when $f$ has irrational multiple root, without loss of generality we can assume that $f(z)=\left(z^{2}+a^{\prime}\right)^{2}\left(z+b^{\prime}\right)$ for certain integers $a^{\prime}, b^{\prime}$ and $a^{\prime} \neq 0$. After change of variables $(x, y, z)=\left(\left(Z^{2}+a^{\prime}\right) X,\left(Z^{2}+a^{\prime}\right) Y, Z\right)$ with the inverse $(X, Y, Z)=(x /$ $\left.\left(z^{2}+a^{\prime}\right), y /\left(z^{2}+a^{\prime}\right), z\right)$ we reduce our problem to the examination of the surface $\mathcal{E}_{f}^{\prime \prime}$ given by the equation

$$
\mathcal{E}_{f}^{\prime \prime}: X^{2}-\left(Z^{2}+a^{\prime}\right) Y^{3}-\left(Z+b^{\prime}\right)=0
$$


Now, we look at the fibration of $\mathcal{E}_{f}^{\prime \prime}$ over the $Y$ line. We consider its generic fibre, which is a curve over the field $\mathbb{Q}(Y)$, and viewing this curve over the extension field $\mathbb{Q}(u)$ of $\mathbb{Q}(Y)$ given by $Y=u^{2}$. The equation of this curve is of the form

$$
C: X^{2}=u^{6} Z^{2}+Z+a^{\prime} u^{6}+b^{\prime} .
$$

Let us note that $C$ is a curve of genus zero with the $\mathbb{Q}(u)$-rational point $P=\left(u^{3}, 0,1\right)$ (it is a point at infinity). So, this is a rational curve over $\mathbb{Q}(u)$. Putting $X=p u^{3}+t, Z=p$ and solving the obtained equation according to $p$ we get the parametrization of our curve

$$
X(t, u)=\frac{u^{3} t^{2}-u+t^{3}\left(a^{\prime} t^{6}+b^{\prime}\right)}{2 u^{3} t-1}, \quad Z(t, u)=\frac{-t^{2}+a^{\prime} u^{6}+b^{\prime}}{2 u^{3} t-1} .
$$

Our reasoning shows that the surface obtained by the base change $Y=u^{2}$ is in fact rational and thus the surface $\mathcal{E}_{f}^{\prime \prime}$ is unirational. This immediately implies that rational points on $\mathcal{E}_{f}$ are dense in the Zariski topology.

(2) Let us put $F(x, y, z)=x^{2}-y^{3}-f(z)$. As in the previous proof we use the method of indetermined coefficients. We look for elements $p, q, r, T \in \mathbb{Q}(s, u)$ such that the curve given by $x=T^{3}+p T^{2}+q T+r, y=T^{2}+s T+u, z=T$ lies on the surface. For $x, y, z$ defined in this way we have

$$
F(x, y, z)=f_{0}+f_{1} T+f_{2} T^{2}+f_{3} T^{3}+f_{4} T^{4}+f_{5} T^{5},
$$

where

$$
\begin{array}{ll}
f_{0}=-d+r^{2}-u^{3}, & f_{1}=-c+2 q r-3 s u^{2} \\
f_{2}=-b+q^{2}+2 p r-3 s^{2} u-3 u^{2}, & f_{3}=-a+2 p q+2 r-s^{3}-6 s u, \\
f_{4}=p^{2}+2 q-3 s^{2}-3 u, & f_{5}=-1+2 p-3 s
\end{array}
$$

Let us notice that the system of equations $f_{3}=f_{4}=f_{5}=0$ has exactly one solution in $\mathbb{Q}(s, u)$ given by

$p=\frac{1+3 s}{2}, \quad q=\frac{-1-6 s+3 s^{2}+12 u}{8}, \quad r=\frac{1+8 a+9 s+15 s^{2}-s^{3}-12 u+12 s u}{16}$.

Substituting these values into the equation $f_{2}=0$ we get

$48 u^{2}-24\left(-3-10 s+s^{2}\right) u-\left(5+32 a-64 b+60 s+96 a s+198 s^{2}+140 s^{3}-3 s^{4}\right)=0$.

Now, solving this equation with respect to $u$ we obtain

$$
u=\frac{-9-30 s+3 s^{2} \pm 4 \sqrt{15 s^{3}+90 s^{2}+9(2 a+5) s+6(a-2 b+1)}}{12} .
$$

Now let us consider the curve $C_{a, b}$ given by the equation

$$
C_{a, b}: v^{2}=15 s^{3}+90 s^{2}+9(2 a+5) s+6(a-2 b+1) .
$$

With the affine change of variables

$$
(s, v)=\left(\frac{X-30}{15}, \frac{Y}{15}\right) \quad \text { with the inverse } \quad(X, Y)=(15(s+2), 15 v)
$$


we transform the curve $C_{a, b}$ into the curve $E_{a, b}$ given by the equation

$$
E_{a, b}: Y^{2}=X^{3}+135(2 a-15) X-1350(5 a+2 b-26) .
$$

We get the following consequence of our reasoning: if infinitely many rational points lie on $E_{a, b}$ then all but finitely many points give us new value $z=T$ (the solution of the equation $\left.f_{0}+f_{1} T=0\right)$ for which the elliptic curve $\mathcal{E}_{f(z)}: x^{2}=y^{3}+f(z)$ has a positive rank. We know that each fibre containing infinitely many rational points is contained in the closure of the set of rational points, say $\mathcal{R}$. We have to show that for all but finitely many $z$ the curve $\mathcal{E}_{f(z)}$ is of positive rank, so the set $\mathcal{R}$ contains infinitely many curves and is therefore two-dimensional. As the surface is irreducible, the set of rational points is dense.

REMARK 2.2. In the first part of Theorem 2.1 we have shown that if the polynomial $f$ has multiple roots then there is a rational change of base $z=\psi(t)$ such that there exists a non-torsion section on the elliptic surface $\mathcal{E}_{f \circ \psi}$. A natural question arises whether it is possible to construct the polynomial $f$ without multiple roots giving a surface $\mathcal{E}_{f}$ with the similar property. It turns out that it is possible to construct demanded polynomials. This is closely connected with the question for which rational numbers $a, b$ the curve $E_{a, b}$ is singular.

The curve $E_{a, b}: Y^{2}=X^{3}+135(2 a-15) X-1,350(5 a+2 b-26)=: g(X) \quad$ is singular if, for the discriminant $\Delta$ of the polynomial $g$, we have

$$
\Delta(a, b)=-16\left(4(135(2 a-15))^{3}+27(1,350(5 a+2 b-26))^{2}\right)=0 .
$$

It is easy to see that it holds if and only if

$$
45(2 a-15)=-t^{2}, \quad 675(5 a+2 b-26)=t^{3},
$$

for a certain rational number $t$. Hence we obtain

$$
a=\frac{-t^{2}+630}{90}, \quad b=\frac{2 t^{3}+75 t^{2}-15,525}{2,700} .
$$

For $a, b$ defined in this way, the curve $E_{a, b}$ is reduced to the form $E: Y^{2}=$ $(X+2 t)(X-t)^{2}$ and the set of its rational points can be parametrized,

$$
X=U^{2}-2 t, \quad Y=U\left(U^{2}-3 t\right) .
$$

Using the above equalities we obtain an explicit form of the rational curve $L$ on the surface $\mathcal{E}_{f}$ in the case when $a, b$ are given by (2.3). We will not, however, present these equations here, since the rational functions defining the curve $L$ are of very high degrees.

Part (1) of our theorem and the above observation suggest the following

Question 2.3. Let $f(z)=z^{5}+a t^{3}+b z^{2}+c z+d$ and let us consider the surface $\mathcal{E}_{f}: x^{2}=y^{3}+f(z)$. What are the conditions that guarantee the existence of a rational base change $z=\psi(t)$ such that there is a non-torsion section on the surface $\mathcal{E}_{f \circ \psi}$ ?

Let us note several interesting corollaries of Theorem 2.1. 
COROLlaRY 2.4. Let $f(z)=z^{5}+a z^{3}+b z^{2}+c z+d \in \mathbb{Z}[t]$ and let us assume that the set of rational points on the curve $E_{a, b}: Y^{2}=X^{3}+135(2 a-15) X-1350(5 a+$ $2 b-26)$ is infinite. Then the diophantine equation $x^{2}-y^{3}-f(z)=t$ has infinitely many solutions in the ring of polynomials $\mathbb{Q}[t]$.

Proof. This observation is an immediate consequence of the reasoning conducted in the proof of the second part of Theorem 2.1. Indeed, by the assumption (curve $E_{a, b}$ has infinite set of rational points) we have shown that for a fixed rational point on $E_{a, b}$ the coefficients of the polynomials $x(T)=T^{3}+p T^{2}+q T+r, y(T)=T^{2}+s T+$ $u, z(T)=T$ are rational and the polynomial $F(x(T), y(T), z(T))$, where $F(x, y, z)=$ $x^{2}-y^{3}-f(z)$, is of degree one. Because the set of rational points on the curve $E_{a, b}$ is infinite, for all but finitely many points from $E_{a, b}(\mathbb{Q})$ we have $f_{1} \neq 0$. Now, solving the equation $f_{0}+f_{1} T=t$, we get that $T=\left(t-f_{0}\right) / f_{1}$. Finally, we obtain the identity

$$
x\left(\frac{t-f_{0}}{f_{1}}\right)^{2}-y\left(\frac{t-f_{0}}{f_{1}}\right)^{3}-f\left(\frac{t-f_{0}}{f_{1}}\right)=t .
$$

This concludes the proof of Corollary 2.4.

COROLlary 2.5. Let $c, d \in \mathbb{Z}$ and $f(z)=z^{5}+c z+d$. Then on the surface $\mathcal{E}_{f}$ the set of rational points is dense in the Zariski topology.

Proof. For $a=b=0$ the equation of the curve $E_{a, b}$ (from the proof of Theorem 2.1) takes the form

$$
E_{0,0}: Y^{2}=X^{3}-2,025 \mathrm{X}+35,100 .
$$

With the assistance of APECS program [2] we have found that $\operatorname{Tors}\left(E_{0,0}\right)$ is trivial and that the rank of the curve $E_{0,0}$ is two. Independent points of infinite order, $P_{1}=(15,90)$ and $P_{2}=(25,10)$, generate the set of rational points on $E_{0,0}$. It follows that the set of rational points on $E_{0,0}$ is infinite.

COROLlary 2.6. Let $a, b, c, d \in \mathbb{Z} \backslash\{0\}$ and consider the surface given by the equation

$$
\mathcal{E}: a x^{2}+b y^{3}+c z^{5}=d .
$$

Then the set of rational points on $\mathcal{E}$ is dense in the Zariski topology.

Proof. After the change of variables $x=X / a^{8} b^{10} c^{12}, y=-Y / a^{5} b^{7} c^{8}, z=$ $-Z / a^{3} b^{4} c^{5}$, the equation of the surface $\mathcal{E}$ takes the form

$$
X^{2}-Y^{3}-Z^{5}=a^{15} b^{20} c^{24} d .
$$

From Corollary 2.5 we get the statement of our theorem.

For instance, taking point $P_{1}=(15,90)$, which is one of the generators of the set $E_{0,0}(\mathbb{Q})$, and performing all necessary calculations presented in the proof of Theorem 2.1 we obtain the identity

$$
\begin{aligned}
& a\left(\frac{25875323 c^{18}+720748 a^{15} b^{20} c^{12} d+8336 a^{30} b^{40} c^{6} d^{2}+64 a^{45} b^{60} d^{3}}{1560896 a^{8} b^{10} c^{15}}\right)^{2} \\
& \quad+b\left(-\frac{87709 c^{12}+1544 a^{15} b^{20} c^{6} d+16 a^{30} b^{40} d^{2}}{13456 a^{5} b^{7} c^{10}}\right)^{3}+c\left(\frac{135 c^{6}+4 a^{15} b^{20} d}{116 a^{3} b^{4} c^{5}}\right)^{5}=d .
\end{aligned}
$$


Note that, in fact, we proved something more. Namely, for every natural number $d$ there is an $S$-integer point on the surface $\mathcal{E}$, where $S=\{p \in \mathbb{P}: p \mid 58 a b c\}$.

3. Rational points on the surface $x^{2}+a y^{5}-z^{6}=b$. In this section we will show that the method employed to prove Theorem 2.1 can be used in another situations. Interested readers can also take a look at paper [10]. We will prove the following

THEOREM 3.1. For every pair $a, b$ of non-zero rational numbers the set of rational points on the surface $\mathcal{S}: x^{2}+a y^{5}-z^{6}=b$ is infinite.

Proof. Our reasoning will be similar to the one presented in the proof of Theorem 2.1. Let us denote $F(x, y, z)=x^{2}+a y^{5}-z^{6}$ and let us put $x=T^{3}+p T^{2}+q T+$ $r, y=u T+v, z=T$. For $x, y, z$ defined in this way we have

$$
F(x, y, z)=f_{0}+f_{1} T+f_{2} T^{2}+f_{3} T^{3}+f_{4} T^{4}+f_{5} T^{5},
$$

where

$$
\begin{array}{lll}
f_{0}=r^{2}+a v^{5}, & f_{1}=2 q r+5 a u v^{4}, & f_{2}=q^{2}+2 p r+10 a u^{2} v^{3}, \\
f_{3}=2 p q+2 r+10 a u^{3} v^{2}, & f_{4}=p^{2}+2 q+5 a u^{4} v, & f_{5}=2 p+a u^{5} .
\end{array}
$$

The system of equations $f_{2}=f_{3}=f_{4}=f_{5}=0$ has exactly three solutions (with respect to $p, q, r, v)$. One of them is defined over $\mathbb{Q}(u)$ (the other two are defined over $\mathbb{Q}(\sqrt{3})(u))$, namely,

$$
p=-\frac{a u^{5}}{2}, \quad q=\frac{3 a^{2} u^{10}}{16}, \quad r=\frac{a^{3} u^{15}}{64}, \quad v=-\frac{a u^{6}}{8} .
$$

Now putting the calculated values to the expressions defining $x, y, z$, we can see that the polynomial $F(x, y, z)$ is of degree one. Solving the equation

$$
F(x(T), y(T), z(T))=b
$$

with respect to $T$ and performing all necessary calculations we obtain the identity

$$
\begin{aligned}
& \left(\frac{118441 a^{18} u^{90}+2^{15} 11863 a^{12} b u^{60}-2^{30} 137 a^{6} b^{2} u^{30}+2^{45} b^{3}}{2^{9} 29^{3} a^{15} u^{75}}\right)^{2} \\
& +a\left(-\frac{9 a^{6} u^{30}-2^{13} b}{58 a^{5} u^{24}}\right)^{5}-\left(\frac{7 a^{6} u^{30}-2^{15} b}{232 a^{5} u^{25}}\right)^{6}=b
\end{aligned}
$$

This concludes the proof of Theorem 2.5.

Using exactly the same reasoning as in the proof of the Theorem 3.1 we get

COROLlary 3.2. Let $a, b, c, d \in \mathbb{Z}, a \neq 0$ and consider the surface given by the equation $\mathcal{S}: x^{2}+a y^{5}+b y-\left(z^{6}+c z\right)=d$. Then the set of rational points on $\mathcal{S}$ is infinite.

\section{REFERENCES}

1. F. Bogomolov and Yu. Tschinkel, On the density of rational points on elliptic fibrations, J. Reine und Angew. Math. 511 (1999), 87-93. 
2. I. Connel, APECS: Arithmetic of plane elliptic curves, 2001, available from http://www. math.mcgill.ca/connell/public/apecs/.

3. G. Faltings, Endlichkeitssätze für abelsche Varietäten über Zahlkörpern, Invent. Math. 73 (1983), 349-366.

4. M. Kuwata and L. Wang, Topology of rational points on isotrivial elliptic surfaces, Int. Math. Research Notices 4 (1993), 113-123. $33-58$.

5. E. Manduchi, Root numbers of fibers of elliptic surfaces, Compos. Math. 99 (1995),

6. Y. I. Manin, Cubic forms: Algebra, geometry, arithmetic, 2nd ed. (North-Holland Publishing, Amsterdam, 1986).

7. R. Munshi, Density of positive rank fibers in elliptic fibrations, J. Number Theory $\mathbf{1 2 5}$ (2007) 254-266.

8. D. E. Rohrlich, Variation of the root number in families of elliptic curves, Compos. Math. 87 (1993), 119-151.

9. J. Silverman, The arithmetic of elliptic curves (Springer-Verlag, New York, 1986).

10. M. Ulas, Rational points on certain elliptic surfaces, Acta Arith. 129 (2007), 167-185. 